\title{
WHY DOES THE SUPERIOR RECTAL ARTERY SKIP THE MIDDLE AND LOWER PARTS OF THE RECTUM BUT SUPPLIES THE UPPER PART OF ANAL CANAL?
}

\author{
Stany W. Lobo ${ }^{1}$, Mangala Kumari ${ }^{2}$, Thejodhar Pulakunta ${ }^{3}$, Sudarshan $\mathrm{S}^{4}$, \\ Rajasekaran Koteeswaran ${ }^{5}$, Tiffany Kurian ${ }^{6}$ \\ ${ }^{1}$ Department of professional and medical education, Meharry Medical College, Nashville, \\ Tennessee, USA \\ ${ }^{2}$ Department of Anatomy, Division of Human Biology, International Medical University, Kuala \\ Lumpur, Malaysia \\ ${ }^{3}$ Department of medical Neuroscience, Dalhousie University, Halifax, Canada \\ ${ }^{4}$ Department of Anatomy, Melaka Manipal Medical College, Manipal Academy of Higher \\ Education, Manipal, India \\ ${ }^{5}$ Department of Pathology/Microbiology, Eastern Virginia Medical School, Norfolk, Virginia, \\ USA \\ ${ }^{6}$ Touro College of Osteopathic medicine, Middletown, New York, USA
}

\section{Dear Editor,}

The rectum is a pelvic organ which follows the sacral curvature. It pierces the pelvic diaphragm and continues as the anal canal in the perineum. The detailed vascular anatomy of the rectum and anal canal is essential to answer relevant clinical questions (Standring, 2020). The pattern of vasculature and lymphatic drainage of the rectum and the anal canal is undisputed and described in most of the standard textbooks of clinical anatomy.

According to Moore et.al. (2006), the rectum and the anal canal is perfused by branches of Inferior mesenteric artery (IMA) and branches of internal iliac arteries (IIA). The superior part of the rectum is perfused by the superior rectal branch of the IMA, whereas the middle and the lower parts are perfused by the branches of IIA through its middle rectal and Internal pudendal branches. However, authors also described the vasculature of anal canal by the combination of both IMA and IIA (Moore et al., 2006). The superior rectal branch of the IMA perfuses the anal canal above the pectinate line and the inferior rectal artery branch of Internal iliac artery perfuses the anal canal below the pectinate line.

According to Richard R. Snell, the lymphatic drainage of superior part of the rectum will follow its vasculature and drain into the inferior mesenteric nodes and the lymph from the rest will drain into the internal iliac nodes. The author also documented that lymphatic drainage of the anal canal above the pectinate line is drained into internal iliac nodes and the rest is to the superficial inguinal nodes (Snell, 2008). The standard textbooks of clinical anatomy describe the abundant anastomoses between branches of IMA and IIA and the portocaval anastomoses at the lower end of the gut.

These descriptions in most textbooks do not provide any reasoning for this unconventional pattern of blood supply. Are there reasons more persuasive than the presence of anastomoses, for why the upper part of the anal canal is supplied by branches of IMA whereas the lower part of the rectum is perfused by branches of IIA? Why does the Superior rectal artery which is a branch of IMA "skip" the middle and lower parts of the rectum and supply the upper part of the anal canal? The embryological territories of vascular distribution of the gut do not support the vasculature of the middle and lower part of the rectum by the branches of IIA.

* Correspondence to: Mangala Kumari.
mangs30@gmail.com


Considering the clinical anatomy of the rectum and anal canal with the emphasis on the prevalence of cancer, occurrence of hemorrhoids and as a site of portocaval anastomoses, we would recommend a revisit to the vasculature of the rectum and anal canal.The topographical location of the vessels and the organs may also recommend this to rectify any errors.

We recommend more focused angiographical and cadaveric studies to accept or to dispute or to find a basis for the current version of vasculature of rectum and anal canal.

\section{References}

Moore KL, Dalley AF, Agur AMR. 2006. Clinically oriented anatomy. Clinically oriented anatomy. p.p-431

Snell RS. 2008. The lower limb. Clinical anatomy by regions, 8, p.p-365.

Standring S. ed., 2020. Gray's anatomy E-Book: the anatomical basis of clinical practice. Elsevier Health Sciences. P.1146-58. 